\title{
Soil microbial trait-based strategies drive metabolic efficiency along an altitude gradient
}

\author{
Jiao Feng ${ }^{1,2,4}$, Xiao-Min Zeng ${ }^{1,2,4}$, Qianggong Zhang ${ }^{3}$, Xin-Quan Zhou ${ }^{1,2}$, Yu-Rong Liu (D) ${ }^{1,2}$ and Qiaoyun Huang ${ }^{1,2}$
}

(c) The Author(s) 2021

Trait-based approaches provide a candidate framework for linking soil microbial community to ecosystem processes, yet how the trade-offs in different microbial traits regulate the community-level metabolic efficiency remains unknown. Herein we assessed the roles of the microbial taxa with particular trait strategies in mediating soil microbial metabolic efficiency along an altitude gradient on the Tibetan Plateau. Results showed that soil microbial metabolic efficiency declined with increasing altitude, as indicated by the increasing metabolic quotient (microbial respiration per unit biomass, $\mathrm{qCO}_{2}$ ) and decreasing carbon use efficiency $(\mathrm{CUE})$. Both $\mathrm{qCO}{ }_{2}$ and CUE were predominantly predicted by microbial physiological and taxonomic attributes after considering key environmental factors including soil $\mathrm{pH}$, substrate quantity and quality. Specifically, the reduced metabolic efficiency was associated with higher investment into nutrient (particularly for phosphorus) acquisitions via enzymes. Furthermore, we identified key microbial assemblies selected by harsh environments (low substrate quality and temperature) as important predictors of metabolic efficiency. These results suggest that particular microbial assemblies adapted to nutrient limited and cold habitats, but at the expense of lower metabolic efficient at higher altitude. Our findings provide a candidate mechanism underlying community-level metabolic efficiency, which has important implications for microbial-mediated processes such as carbon dynamics under global climate changes.

ISME Communications; https://doi.org/10.1038/s43705-021-00076-2

\section{INTRODUCTION}

Soil microorganisms are critical drivers of the global carbon $(C)$ cycle because of their roles in both soil organic $C$ (SOC) decomposition and formation, regulating major $C$ flux between soil and atmosphere $[1,2]$. Soil $C$ stock is determined by the balance between microbial organic matter decomposition and biomass build-up [3]. Therefore, the metabolic efficiency of microbial community such as metabolic quotient (microbial respiration per unit biomass, $\mathrm{qCO}_{2}$ ) and $\mathrm{C}$ use efficiency (CUE) are fundamental for the rates of ecosystem $C$ storage [3-5]. Recent studies suggest that the inclusion of metabolic efficiency into existing models improves the prediction of soil $\mathrm{C}$ cycling under global changes [6, 7]. The metabolic efficiency of microbial communities can vary with environmental conditions, and are known to be influenced by abiotic factors (primarily climates and substrate quality) [4, 8]. Moreover, previous studies indicated that altered environmental conditions also induced changes in microbial community structure and physiological attributes $[9,10]$, which may also contribute to changes in communitylevel metabolic efficiency [11]. However, despite the acknowledgment that intrinsic properties of microbial community are critical to ecosystem functions $[12,13]$, how these multiple physiological traits inherent to complex microbial community regulate their energy efficiency remains unclear.
A recent trait-based conceptual framework suggests that the trade-offs between multiple physiological traits play fundamental roles in governing soil C cycling under environmental changes [3]. Microbial community is assumed to be inefficient in soils with low substrate quality (i.e., higher soil C:N and C:P ratio) $[8,14]$, as physiological traits related to resource acquisition (e.g., enzyme secretion) are energetically expensive [15]. Moreover, harsh environments like extreme low temperature and drought could also reduce microbial growth rate and enhance the $C$ cost of maintenance $[11,16]$. The trade-offs between different traits could potentially influence the partition of detrital $C$ into biomass production vs. maintenance respiration, causing changes in metabolic efficiency of microbial community as a whole, with important implications for $\mathrm{C}$ cycling under global climate changes.

Given that different microbial taxa have distinct physiological trait combinations, community-level physiological changes may be a consequence of shifts in the relative abundance of specific taxa $[11,17]$. Interpreting microbial taxonomic composition under the concept of trait-based ecology may hint at a way to further clarify the underlying mechanisms for regulating community-level metabolic quotient in the context of next-generation sequencing and big data. This topic was moved forward by grouping microbial taxa into different life-history strategies according to their growth vs. adaptation strategies, such as $r$ - (fast-growing opportunistic

\footnotetext{
${ }^{1}$ State Key Laboratory of Agricultural Microbiology, Huazhong Agricultural University, Wuhan 430070, China. ${ }^{2}$ College of Resources and Environment, Huazhong Agricultural University, Wuhan 430070, China. ${ }^{3}$ Key Laboratory of Tibetan Environment Changes and Land Surface Processes, Institute of Tibetan Plateau Research, Chinese Academy of Sciences, Beijing 100101, China. ${ }^{4}$ These authors contributed equally: Jiao Feng, Xiao-Min Zeng. ${ }^{凶}$ email: yrliu@mail.hzau.edu.cn
}

Received: 23 August 2021 Revised: 18 November 2021 Accepted: 23 November 2021

Published online: 03 December 2021 
species) and $K$-strategists (slow-growing equilibrium species) [18] and the further defined Yield (growth yield)-Acquisition (resource acquisition)-Stress (stress tolerance) strategies [3]. For instance, $\beta$ Proteobacteria and Bacteroidetes are commonly classified as $r$ strategists, while Acidobacteria are $K$-strategists [18, 19]. However, the classification based on high phylogenetic levels (such as at the order or phylum levels) should be used with caution, because microbial taxa with similar functions are commonly phylogenetically diverse and taxonomically associated strains may also have different physiological traits $[20,21]$. Microbial community could also be grouped into different functional assemblies based on the co-occurrence or association patterns from networks, offering new insights into complex microbial community structure and soil functioning $[10,22]$. Yet, we know little about the effects of shifts in microbial assemblies with different functional traits on the metabolic efficiency of microbial community.

Altitude gradient provides a "natural" setting to test the effects of environmental changes on ecosystem processes, because of the drastic changes in climate, biotic and abiotic attributes over short distances within a landscape. Previous studies have reported that low temperature at higher altitude commonly leads to accumulation of soil organic matter with low substrate quality (i.e., higher soil C:N and C:P ratios) [23]. Moreover, soil microbial community composition shifted significantly along altitude gradients [9]. We assumed that (1) the metabolic efficiency of microbial community would be low at higher altitude due to the likely higher energy investments into resource acquisition; (2) the shifts in microbial assemblies with distinct trait strategies may contribute to changes in community-level metabolic efficiency. To test our hypotheses, we collected soil samples from 28 sites along an altitude gradient on the Tibetan Plateau. This region is the highest and largest plateau and has been declared as the "Third Pole" due to its harsh alpine environments [24], providing ideal platform for exploring the trade-offs between different physiological trait-based strategies. We investigated the associations between microbial metabolic efficiency (including $\mathrm{qCO}_{2}$ and CUE) and resource acquisition strategies (soil enzymes and ecoenzymatic stoichiometric ratios). Furthermore, we conducted network analysis to explore key microbial functional assemblies that affect the community-level physiological traits and metabolic efficiency.

\section{MATERIALS AND METHODS \\ Site and sampling}

This study was conducted along an altitude gradient (ranging from 2974 to $3558 \mathrm{~m})$ at Mount Segrila $\left(29^{\circ} 34^{\prime}-29^{\circ} 37^{\prime} \mathrm{N}, 94^{\circ} 19^{\prime}-94^{\circ} 22^{\prime} \mathrm{E}\right)$ on the southeastern part of the Tibetan Plateau (Fig. S1). The mean annual temperature (MAT) declines significantly $\left(R^{2}=0.989\right)$ from approximately 8.6 to $-0.7^{\circ} \mathrm{C}$ as the altitude increased, with a decreasing rate of temperature at $0.54-0.73^{\circ} \mathrm{C}$ per $100 \mathrm{~m}$ [25, 26]. The mean annual precipitation (MAP) ranges between 680 and $1134 \mathrm{~mm}$, with over $80 \%$ of the precipitation occurring during the growing season (between May to September) [25]. The MAT and MAP at the elevation above $3100 \mathrm{~m}$ are less than $4.2^{\circ} \mathrm{C}$ and more than $1000 \mathrm{~mm}$, respectively, indicating that temperature instead of water availability is the dominant growth-limiting factor for biology [27, 28]. Both microbial biomass and the associated functions were maximum during the growing season [29]. The surface soils begin to freeze at the end of October and start to thaw at the beginning of the next March [25]. The dominant vegetation types shift from temperate coniferous and broadleaved mixed forests to frigid dark coniferous forests as the altitude increased [9]. The predominant soil type is Luvisols and Cambisols according to World Reference Base for Soil Resources [30].

Twenty-eight representative sites were selected in August 2018, covering the dominant vegetation types along the altitude gradient. At each site, five $1 \mathrm{~m} \times 1 \mathrm{~m}$ sub-plots were located at each corner and the center of a $50 \mathrm{~m} \times 50 \mathrm{~m}$ area. Five replicate soil samples $(0-10 \mathrm{~cm})$ were collected from the understory or adjacent open grasslands in each site. After removing visible stones, roots and plant materials, collected soil samples were homogenized. Soil samples were kept on ice when transporting to the laboratory and then divided into two sub-samples.
One subsample was stored at $-20^{\circ} \mathrm{C}$ for the analyses of microbial community (i.e., DNA extraction and MiSeq Illumina sequencing). The other subsample was stored at $4{ }^{\circ} \mathrm{C}$ for the analyses of biological activities such as enzyme activities and microbial metabolic efficiency.

\section{Measurement of soil chemical properties}

Soil $\mathrm{pH}$ was measured with a glass electrode with a 1:2.5 soil/distilled water ratio. $\mathrm{SOC}$ was determined by $\mathrm{K}_{2} \mathrm{CrO}_{7}$ oxidation titration method [31]. Soil total $\mathrm{N}(\mathrm{TN})$ was quantified by an elemental analyzer (Vario PYRO Cube, Elementar, Germany). Soil total P (TP) was measured using a digestion method [32]. Soil dissolved organic $\mathrm{C}$ and $\mathrm{N}$ were extracted with deionized water at a ratio of 1:4 (w/v) and determined using a TOC analyzer (vario TOC, Elementar, Germany). Soil $\mathrm{NH}_{4}{ }^{+}$and $\mathrm{NO}_{3}{ }^{-}$were determined by a colorimetric method using a continuous flow analyzer (AA III, BRAN + LUEBBE $\mathrm{GmbH}$, Germany) after being extracted with $2 \mathrm{M} \mathrm{KCl}$. Soil available $P$ was measured by the Olsen bicarbonate method [33].

\section{Analysis of soil bacterial and fungal communities}

Soil DNA was extracted using the MoBio PowerSoil DNA Isolation Kit (MoBio Laboratories, Carlsbad, CA, USA) according to the manufacturer's instructions. The diversity and composition of bacterial and fungal communities were measured by amplifying the V3-V4 region of the $16 \mathrm{~S}$ rRNA gene with primer pairs 338F/806R [34], and the ITS gene with primers ITS1F/ITS2R [35], respectively. The purified amplicons were mixed equimolarly, and $2 \times 300$ bp paired-end sequencing was conducted using an Illumina Miseq sequencer (Illumina Inc., San Diego, USA). We used the UPARSE approach to cluster Operational Taxonomic Units (OTUs or phylotypes) with a picking strategy at $97 \%$ sequence similarity. The taxonomic information for bacterial and fungal OTUs were assigned using the SILVA and Unite ribosomal RNA gene database, respectively [36]. The a-diversity (i.e., Shannon index) and community composition for bacteria and fungi were calculated based on $97 \%$ OTU similarity of obtained sequences. Amplicon sequencing approach has been widely applied to characterize microbial community composition, although some identified taxa may be inactive or dormant $[34,35]$.

\section{Analysis of the putative microbial physiological traits}

Physiological traits involved in microbial resource acquisitions were assessed by potential extracellular enzyme assays [3]. All enzyme measurements were conducted using sieved soil $(<2 \mathrm{~mm})$ at field moisture within one week after sampling. Soil extracellular enzyme activities related to $C$ ( $\alpha-1,4$-glucosidase $[A G], \beta-1,4$-glucosidase $[B G]$, cellobiohydrolase $[\mathrm{CBH}]$ and xylanase $[\mathrm{XYL}]$ ), $\mathrm{N}$ ( $\mathrm{N}$-acetyl- $\beta$-D-glucosaminidase [NAG] and leucine aminopeptidase [LAP]) and $\mathrm{P}$ (acid phosphatase [AP]) acquisitions were measured by fluorimetric methods [37]. Briefly, $0.50 \mathrm{~g}$ of fresh soil was mixed with $50 \mathrm{~mL}$ of deionized water and stirred vigorously using a magnetic stir plate for $15 \mathrm{~min}$. Then, $50 \mu \mathrm{L}$ of soil homogenate, $100 \mu \mathrm{L}$ of fluorometric substrate solution $\left(200 \mu \mathrm{mol} \mathrm{L}^{-1}\right)$ and $50 \mu \mathrm{L}$ of acetate buffer $\left(0.2 \mathrm{~mol} \mathrm{~L}^{-1}, \mathrm{pH} 5.5\right)$ were mixed and incubated at $30^{\circ} \mathrm{C}$ for $3 \mathrm{~h}$ [37]. The released fluorescence was measured using a multifunctional microplate reader (Tecan Spark ${ }^{\mathrm{TM}} 10 \mathrm{M}$, Männedorf, Switzerland) at $360 \mathrm{~nm}$ excitation and $450 \mathrm{~nm}$ emission wavelengths. Extracellular enzyme activities were expressed as $\mathrm{nmol} \mathrm{h} \mathrm{h}^{-1} \mathrm{~g}^{-1}$ soil.

Additionally, specific enzyme activities were calculated by normalizing activities to units per mg MBC, in order to avoid the variations induced by biomass change. Ecoenzymatic vector length (Vlength, relative C: nutrientacquiring ratio) and angle (Vangle, relative $\mathrm{P}: \mathrm{N}$-acquiring ratio) created by the plot of proportional enzymatic $C: N$ and $C: P$ ratios were further calculated to illustrate the relative microbial resource acquisition strategies [38]. Higher Vlength indicates relatively higher $C$ vs. nutrient acquisition strategies, and higher Vangle suggests higher $\mathrm{P}$ vs. $\mathrm{N}$ acquisition efforts. Soil Vlength and Vangle were calculated using Eqs. (1) and (2), respectively:

Vlength $=\sqrt{x^{2}+y^{2}}$

Vangle $($ degree $)=$ degrees $(\operatorname{atan} 2(x, y))$

where atan2 represents the four-quadrant inverse tangent, $x$ and $y$ represent the relative $C: P$ and $C: N$ acquiring enzyme ratios, respectively.

\section{Evaluations of metabolic efficiency}

Microbial metabolic efficiency was evaluated using both $\mathrm{qCO}_{2}$ and $\mathrm{CUE}$, in which lower $\mathrm{qCO}_{2}$ and higher CUE indicate higher metabolic efficiency 
$[5,39]$. Microbial respiration was estimated by an incubation method $[40,41]$. The soils were incubated aerobically in $250 \mathrm{~mL}$ incubation bottles at $20^{\circ} \mathrm{C}$ for 14 days. We chose $20^{\circ} \mathrm{C}$ for incubation according to the averaged ground temperature in the growing season along the altitude gradient [25]. The short-term (14 days) aerobic incubations were selected to minimize the effects of changes in soil labile substrate and microbial community on soil respiration [42]. The bottles were sealed using parafilms with small holes that enable the exchange of gas, but minimized evaporation and soil water loss [40]. During incubation, $60 \%$ of water holding capacity was maintained by weighting the incubation bottles regularly and adding distilled water to compensate for water loss. The bottles were hermetically closed during the $2 \mathrm{~h}$ sampling period, and the concentrations of respired $\mathrm{CO}_{2}$ were measured using a gas chromatography (Agilent 7890A, Agilent Technologies, USA). The rate of soil respiration was quantified as $\mathrm{mg} \mathrm{CO}-\mathrm{CO}_{2}^{-1}$ dry soil $\mathrm{h}^{-1}$. Soil microbial biomass $C(\mathrm{MBC})$ was determined by a chloroform fumigation-extraction

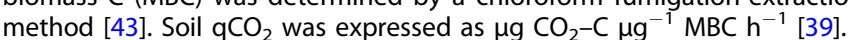

Microbial CUE was calculated indirectly using the biogeochemicalequilibrium model (Eqs. (3-5)) [44, 45]:

$C U E=C U E_{\max } \times \sqrt{\frac{S_{C: N} \times S_{C: P}}{\left(K_{C: N}+S_{C: N}\right) \times\left(K_{C: P}+S_{C: P}\right)}}$

$S_{C: N}=B_{C: N} / L_{C: N} \times 1 / E E A_{C: N}$

$S_{C: P}=B_{C: P} / L_{C: P} \times 1 / E_{C: P}$

In these functions, EEA $\mathrm{C}_{\mathrm{N} N \mathrm{~N}}$ was calculated as $\ln (\mathrm{BG}) / \ln (\mathrm{NAG}+\mathrm{LAP})$, and $E_{C: P}$ was calculated as $\ln (B G) / \ln (A P)$. Molar C:X ratios of labile substrates were used to estimate $L_{C: N}$ and $L_{C: P}$. Labile $C, N$ and $P$ were DOC, inorganic $\mathrm{N}$ (the sum of $\mathrm{NH}_{4}{ }^{+}$and $\mathrm{NO}_{3}{ }^{-}$) and available $\mathrm{P}$, respectively. $\mathrm{B}_{\mathrm{C}: \mathrm{N}}$ and $\mathrm{B}_{\mathrm{C}: \mathrm{P}}$ were the molar ratios of microbial biomass $C: N$ and $C: P$, respectively. $\mathrm{CUE}_{\max }$ was fixed to 0.60 and represented maximum microbial growth efficiency according to thermodynamic constraints based on the saturating Michaelis-Menten formulation. $K_{c: x}$ was the half-saturation constant $(0.50)$.

\section{Statistical analysis}

We first identified the patterns of $\mathrm{qCO}_{2}, \mathrm{CUE}$ and microbial resource acquisition strategies (including specific enzyme activities, Vlength and Vangle) along the altitude gradient. Principal coordinate analysis (PCoA) was conducted to evaluate variations in the taxonomic composition of bacterial and fungal community. Statistical differences in the microbial community composition were tested using the permutational multivariate analysis of variance (PERMANOVA) by "vegan" package in R 4.0.2 (http:// cran.r-project.org/). We conducted a Random Forest analysis to identify the statistically significant predictors of $\mathrm{qCO}_{2}$ and CUE using the "rfPermute" package [46]. We compared the percentage increases in the mean squared error (i.e., \%IncMSE) to evaluate the relative importance of different variables in predicting $\mathrm{qCO}_{2}$ and CUE.

We further conducted a co-occurrence network and identified main ecological clusters (modules or assemblies) of strongly correlated OTUs. To reduce rare OTUs, we removed OTUs with relative abundances less than $0.01 \%$ of bacterial and fungal sequences, respectively [22]. The cooccurrence network was inferred according to the Spearman correlation matrix calculated using the "WGCNA" package. The nodes in networks represent OTUs and the edges connecting different nodes represent correlations between OTUs. To reduce false positive results, we adjusted all $P$-values for multiple correlations using Beniamini and Hochberg false discovery rate (FDR) [47]. Robust correlations with the Spearman correlation coefficients $>0.60$ and FDR adjusted $P$-values $<0.01$ were selected to construct the co-occurrence networks. The network was visualized with Gephi (http://gephi.github.io/). The relative abundance of each module was calculated by averaging the standard relative abundances (z-score) of all taxa belonged to each module [48].

To identify essential microbial assemblies affecting $\mathrm{qCO}_{2}$ and microbial physiological traits, we further conducted a Random Forest analysis by incorporating all bacterial and fungal modules into the model. The relationships between the relative abundances of key microbial modules and environmental factors and microbial physiological traits were evaluated using Spearman's rank correlation analysis. Similarly, we used Spearman's rank correlation analysis to evaluate the relationships of different genus in key microbial modules with $\mathrm{qCO}_{2}, \mathrm{CUE}$ and microbial physiological traits, in order to identify major taxa driving the linkages between $\mathrm{qCO}_{2}$ and different physiological traits.

\section{RESULTS}

Microbial metabolic efficiency and resource acquisition traits along the altitude gradient

Microbial metabolic quotient $\left(\mathrm{qCO}_{2}\right)$ at a community level increased $(P<0.01)$, while CUE decreased with increasing altitude $\left(P<0.05\right.$, Fig. 1a). SOC, soil $\mathrm{NH}_{4}{ }^{+}$and $\mathrm{NO}_{3}{ }^{-}$increased significantly with increasing altitude, while there were no significant changes in soil TP and available $P$ as the altitude increased. Soil C:N:P stoichiometric ratios shifted significantly, with soil C/N, C/P and N/ $P$ ratios all increased with increasing altitude (Fig. 2b). Likewise, there were significant shifts of microbial resource acquisition strategies toward higher investments in nutrients compared to $C$ (i.e., declining Vlength) with increasing altitude (Fig. 1b). In particular, specific AP activities that standardized by per unit MBC enhanced significantly as the altitude increased (Fig. 2b). The relative $\mathrm{P}$ vs. $\mathrm{N}$-acquiring enzyme activities (i.e., Vangle) was generally higher than $45^{\circ}$, but did not varied with increasing altitude (Fig. 1b). The specific enzyme activities associated with $C$ (including $A G, B G, C B H$ and $X Y L$ ) and $N$ (including NAG and LAP) acquisitions showed no significant variations along the altitude gradient (Fig. 2b).

\section{Linking biotic and abiotic factors to metabolic efficiency}

Both Random Forest and correlation analysis consistently showed significant associations of microbial taxonomic and physiological traits with metabolic efficiency (Fig. 2). Shifts in microbial resource acquisition strategies (including Vlength and Vangle) and microbial community composition were essential predictors of $\mathrm{qCO}_{2}$, after considering essential abiotic attributes including soil $\mathrm{pH}$, substrate quantity and quality (Fig. 2a). Regression analysis further indicated that $\mathrm{qCO}_{2}$ correlated negatively with Vlength and specific $\mathrm{N}$-acquiring enzyme activities (Fig. 2b). Likewise, CUE could also be predicted by microbial taxonomic and physiological attributes, including the first dimensional PCOA ordination of bacterial community (B_PCoA1), Vlength and specific P-acquiring enzyme activities (Fig. 2). Soil CUE showed positive relationships with Vlength, but negative associations with specific P-acquiring enzyme activities. In addition, soil abiotic factors such as SOC, TP, $\mathrm{C} / \mathrm{N}, \mathrm{C} / \mathrm{P}$ and available $\mathrm{P}$ were also significant predictors of variations in metabolic efficiency. Soil $\mathrm{qCO}_{2}$ was positively related to SOC, soil $\mathrm{NH}_{4}{ }^{+}, \mathrm{NO}_{3}{ }^{-}$concentrations and soil $\mathrm{C} / \mathrm{N}, \mathrm{C} / \mathrm{P}$ and $\mathrm{N} / \mathrm{P}$ ratios. In contrast, CUE showed overall negative associations with soil stoichiometric ratios (including soil $\mathrm{C} / \mathrm{N}, \mathrm{C} / \mathrm{P}$ and N/P ratios), but positive relationships with soil total $P$ and available $P$ (Fig. 2).

\section{Specific microbial taxa drive changes in physiological traits and metabolic efficiency}

There were significant shifts in taxonomic composition for bacterial community along the altitude gradient (PERMANOVA, $P<0.05$; Fig. S2). The relative abundance of Proteobacteria and Acidobacteria increased, while that of Cyanobacteria declined with increasing altitude (Table S1). Other dominant bacterial phyla, including Actinobacteria, Chloroflexi, Bacteroidetes, Gemmatimonadetes and Firmicutes, were independent of altitude $(P>0.05$; Table S1), although some of these phyla showed significant associations with soil abiotic factors (Fig. S3). The overall fungal community composition and the relative abundances of all the dominant fungal phyla, including Ascomycota, Basidiomycota and Mortierellomycota, showed no significant variations along the altitude gradient (Table S1; Fig. S2). Among these microbial phyla that varied with altitude, only Proteobacteria and Cyanobacteria had significant associations with microbial physiological traits and metabolic efficiency (Table S2). 

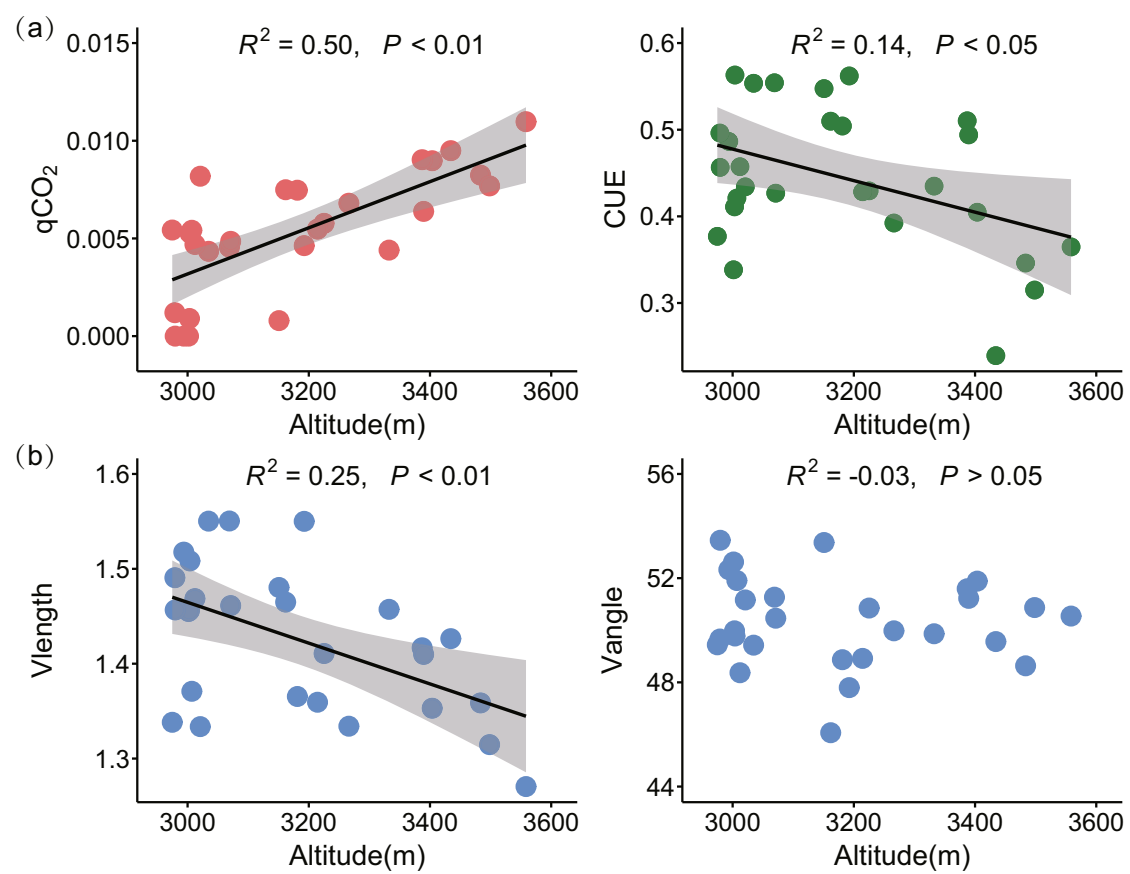

Fig. 1 Patterns of microbial metabolic efficiency and resource acquisition strategies along the altitude gradient. a Metabolic efficiency,

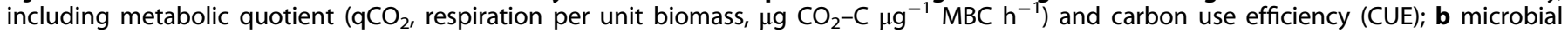
resource acquisition strategy. The Vlength quantified the relative $\mathrm{C}$ vs. nutrient $(\mathrm{N}$ and $\mathrm{P}$ )-acquiring enzyme activities, and lower Vlength indicated relative higher nutrient acquisition strategies (relative to $\mathrm{C}$ ); the Vangle quantified the relative $\mathrm{P}$ vs. $\mathrm{N}$-acquiring enzyme activities, and higher Vangle represented relatively higher $\mathrm{P}$ vs. $\mathrm{N}$ acquisition strategies.

In contrast, results of network analyses showed significant changes in the relative abundance of different microbial assemblies (clusters of microbial phylotypes that highly correlated with each other) along the altitude (Figs. 3 and S4). The soil bacterial and fungal phylotypes could be grouped into four (B_Mod\#0-3) and eight (F_Mod\#0-7) major microbial assemblies, respectively. Over these assemblies, the abundances of phylotypes belonging to B_Mod\#0 and F_Mod\#6 increased significantly with increasing altitude, while those belonging to B_Mod\#2 and F_Mod\#1 exhibited opposite patterns (Fig. S4). More importantly, these shifted bacterial and fungal assemblies with altitude were essential predictors of the variations in Vlength, $\mathrm{qCO}_{2}$ and $\mathrm{CUE}$, even considering multiple edaphic properties (Figs. 3 and S5). Specifically, the relative abundance of B_Mod\#0 was negatively related to Vlength and $\mathrm{CUE}$, but positively to $\mathrm{qCO}_{2}$ and P-acquiring enzyme activity (Figs. 3 and S4). On the contrary, the relative abundance of $B \_$Mod\#2 showed totally reversed relationships with these potential resource acquisition strategies and metabolic efficiency. For fungal community, the relative abundance of $F \_$Mod\# 1 showed generally positive relationships with Vlength and CUE, but negatively with $\mathrm{qCO}_{2}$ and $\mathrm{P}$-acquiring enzyme activity. The proportion of $\mathrm{F} \_$Mod\#6 correlated positively with $\mathrm{qCO}_{2}$ and enzyme activities involved in $\mathrm{C}, \mathrm{N}$ and $\mathrm{P}$ acquirements (Figs. 3 and S4).

The proportion of most genera within B_Mod\#0 and F_Mod\#6 increased with increasing altitude, which also showed negative relationships with Vlength and CUE, but positive relationships with $\mathrm{qCO}_{2}$ (Fig. 4b). In contrast, the proportions of most genera within B_Mod\#2 and F_Mod\#1 declined as the altitude increased, and correlated positively with Vlength and CUE, but negatively with $\mathrm{qCO}_{2}$. The key assemblies for bacterial community were dominated by Proteobacteria, Actinobacteria, Acidobacteria and Chloroflexi (Fig. 4a). Most of the genera within B_Mod\#0 belonged to the Proteobacteria (57 out of 125) and Acidobacteria (18 out of 125) (Table S2). For instance, genera Bryobacter, GAS113, Edaphobacter, Roseiarcus, and Granulicella all showed significantly positive associations with altitude and $\mathrm{qCO}_{2}$, but negatively with Vlength and CUE (Table S2). The majority of genera within B_Mod\#2 belonged to the Proteobacteria (77 out of 233) and Actinobacteria (67 out of 233). In particular, genera such as Sphingomonas, Nordella, Microlunatus, Rhizobacter and Lysobacter, all showed negative relationships with altitude and $\mathrm{qCO}_{2}$, but positive correlations with Vlength and CUE. For fungal community, most of the phylotypes related to altitude were within Ascomycota, with Coniochaeta, Neurospora, Epicoccum and Mortierella etc. as essential genera predicting Vlength and metabolic efficiency (Table S2).

\section{DISCUSSION}

Our study established empirical associations between shifts in microbial community composition with different trait combinations and metabolic efficiency, accounting for internal controls over microbial metabolic efficiency and the associated ecosystem functions. We show that increasing $C$ cost of maintenance (i.e., $\mathrm{q}\left(\mathrm{O}_{2}\right)$ and decreasing CUE was linked to increasing nutrient (particularly P) acquisition strategies along the altitude gradient. These results indicate that in soils with lower substrate quality (higher C:N and C:P ratios), resource limitations drive microbial communities to invest more energy into resource acquisition strategies that trade-off against growth yield at higher altitude. Such a trade-off of physiological traits could be related to shifts in microbial taxa with distinct trait-based strategies. In particular, the shifted bacterial and fungal assemblies with altitude were also essential predictors of microbial resource acquisition strategies and metabolic efficiency. Based on the framework of trait-based ecology, our data provide evidence for the linkages between shifts in microbial community composition and community-level metabolic efficiency along a broad environmental gradient. These findings illustrate that the intrinsic properties of microbial community play crucial roles in mediating the efficiency of microbial processes such as CUE, highlighting that the 


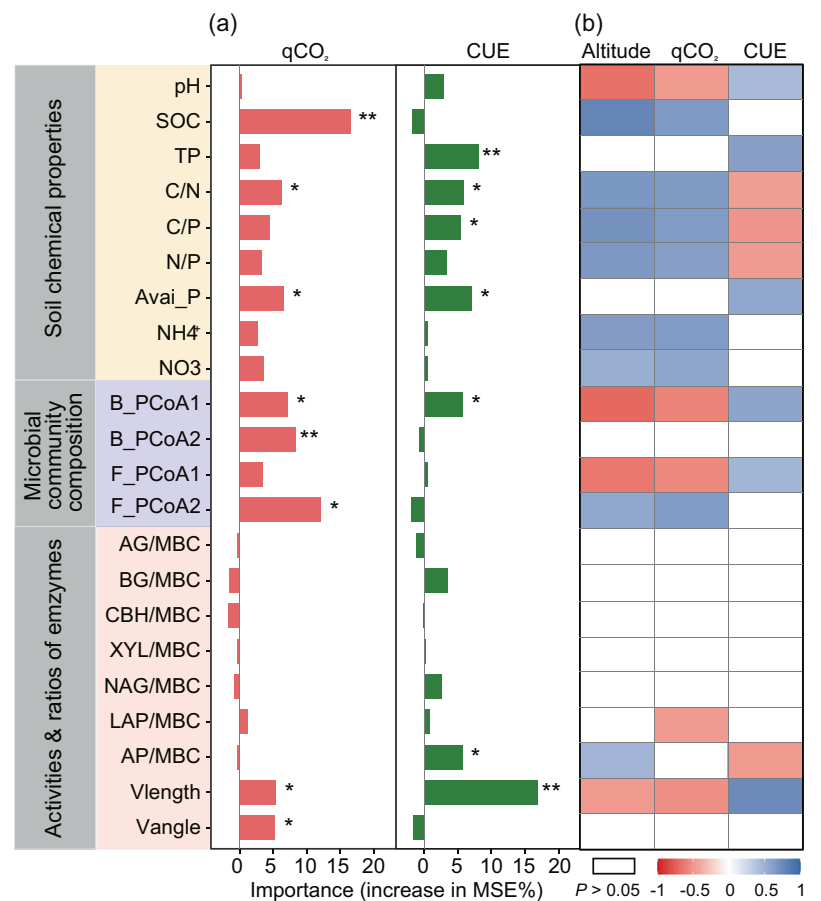

Fig. 2 Major predictors of soil metabolic efficiency along the altitude gradient. a The percentage increases in the mean squared error (\%IncMSE) based on Random Forest analysis; $\mathbf{b}$ relationships of metabolic quotient $\left(\mathrm{qCO}_{2}\right)$ and carbon use efficiency (CUE) with selected biotic and abiotic attributes. SOC, soil organic C; TP, total $P$; Avai_P, available P; B_PCoA1, B_PCoA2, F_PCoA1 and F_PCoA2 were the two dimensional PCoA ordination of taxonomic composition for bacterial and fungal community, respectively. AG, $\alpha-1,4$-glucosidase activities; $B G, \beta-1,4$-glucosidase; $C B H$, cellobiohydrolase; $X Y L$ xylanase; NAG, N-acetyl- $\beta$-D-glucosaminidase; LAP, leucine aminopeptidase; AP, acid phosphatase. Enzyme/MBC represents specific enzyme activities calculated by normalizing activities to units $/ \mathrm{mg}$ MBC. ${ }^{*} P<0.05 ;{ }^{*} P<0.01$.

incorporation of intrinsic microbial properties into models is important to better predict biogeochemical cycles and their feedbacks to climate changes.

Consistent with our first hypothesis, the metabolic efficiency of microbial community was low (higher $\mathrm{qCO}_{2}$ and lower CUE) at higher altitude, partly due to the higher energy investments in nutrient acquisition strategies (Fig. 1; Table S1). At higher altitude with lower substrate quality (higher soil C:N and C:P ratios), the lower Vlength and higher specific P-acquiring enzyme activities indicate that more energy are allocated to acquire the most limited nutrients ( $\mathrm{N}$ and $\mathrm{P}$ ) under unbalanced resource stoichiometry [15]. The production of extracellular enzymes to acquire nutrients from complex biomolecules is energetically expensive, and thus may contribute to higher $C$ cost of maintenance and lower CUE at higher altitude [3]. In support of this, we found that the specific P- acquiring enzyme activities correlated negatively with CUE (Fig. 2), indicating that increasing microbial trait strategies of resource $(P)$ acquisition through enzymes reduced metabolic efficiency at higher altitude. Similar relative nutrient $(\mathrm{N}$ and P) constraints to microbial metabolism were also predicted in high-latitude grasslands with low temperature using a new enzymatic stoichiometric model [49]. Our results provide evidence for a clear trade-off between community-level resource acquisition potential and growth yield based on empirical relationships along a highly heterogeneous environmental gradient.

Our study also presents that a large amount of variation in metabolic efficiency was explained by altitude, which correlated highly $\left(R^{2}=0.989\right)$ with temperature in these harsh alpine environments $[25,26]$. Therefore, it is plausible that growth traits also trade-off with cold-stress tolerance strategies along the altitude gradient in the highest plateau on Earth. Many previous studies suggested that low temperature could inhibit the growth and metabolic activity of microorganisms [50,51]. Traits involved in cold adaptions such as biofilm formation and membrane modifications were favored under cold environments [52, 53], which may contribute to the lower metabolic efficiency under cold conditions. As such, the harsh environments (low substrate quality and temperature) may induce trade-offs among multiple strategies including growth yield, resource acquisition and stress tolerance. Future works on physiological adaptions of microbial communities to cold-stress tolerance and their effects on microbial metabolic efficiency will consolidate our findings.

Additionally, microbial taxonomic variations were highlighted as essential predictors of metabolic efficiency according to our results of Random Forest analysis (Fig. 2). This supports previous studies that community-level variations in physiological traits mostly reflect the legacy effects of long-term environmental changes on microbial community composition [12]. In this study, soil samples were taken in the last month of the growing season, when soil availability is high and the immediate freeze-thawing effects can be disregarded. As such, our study could provide a snapshot of shifts in microbial community composition and trait strategies across a low temperature gradient with distinct changes in abiotic factors (including soil $\mathrm{pH}$, substrate quantity and quality). Soils at higher altitude are likely to host microbial taxa that adapted to low substrate quality and temperature [54, 55]. In support of this, we observed increased abundance of Proteobacteria with increasing altitude, which is always dominant in cold and oligotrophic conditions [52, 56, 57]. Furthermore, the relative abundance of Proteobacteria associated positively with the potential functions involved in nutrient acquisition (i.e., lower Vlength) and $\mathrm{qCO}_{2}$ (Table S1), indicating that environmental selection of specific taxa contribute to community-level changes in physiological traits and metabolic efficiency [12]. However, except for Proteobacteria, most dominant bacterial and fungal phyla were independent of altitude and acted as weak predictors of changes in physiological traits and metabolic efficiency. These results indicate that microbial classification based on higher phylogenetic levels (such as at the order or phylum levels) have limitations in explaining community-level physiological strategies, because taxonomically related strains can have divergent features, while dissimilar strains may exhibit identical traits $[20,21]$.

Microbial community could also be grouped into assemblies with particular trait combinations based on different cooccurrence or association patterns, offering new insights into complex microbial community structure and soil functioning $[10,22]$. Microbial assemblies within co-occurrence network are significant predictors of microbial physiological traits, even considering other key environmental attributes (Figs. 3 and S5). In particular, the relative abundance of $B \_M o d \# 0$ increased significantly with increasing altitude (concurrent declining temperature) and soil C:N and C:P ratios (Fig. S4), suggesting that these taxa adapted to low temperature and substrate quality at higher altitude. Interestingly, these adapted taxa within B_Mod\#0 also showed significant associations with community-level microbial nutrient acquisition traits, $\mathrm{qCO}_{2}$ and $\mathrm{CUE}$, further indicating that environmental selection of specific taxa drives changes in physiological traits and the metabolic efficiency of microbial community. Most of genera within B_Mod\#0 were from Alphaproteobacteria and Acidobacteria, which were mostly $K$-strategists (slow-growing equilibrium species) and also the main sources of assumed cold-adaption genes such as those encoding OstA (trehalose phosphate synthase), OstB (trehalose phosphatase) and Fatty acid desaturases etc. [52]. In contrast, B_Mod\#2 may be mainly composed of microbial groups with low maintenance efforts and weak adaption to low temperature and nutrient 
(a)

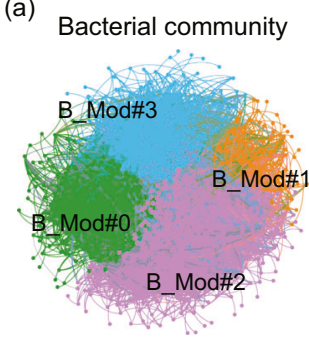

(c)
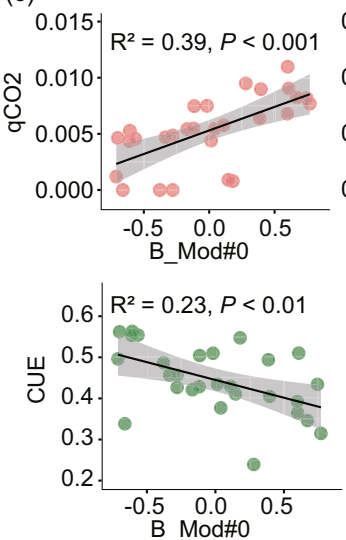

Fungal community

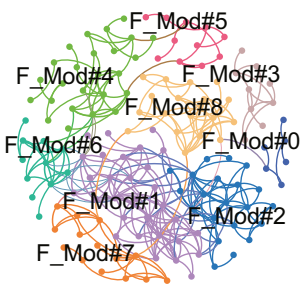

0.01
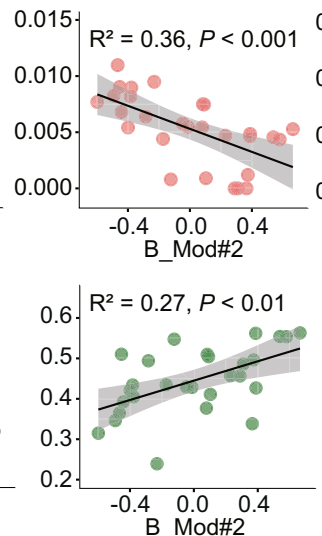

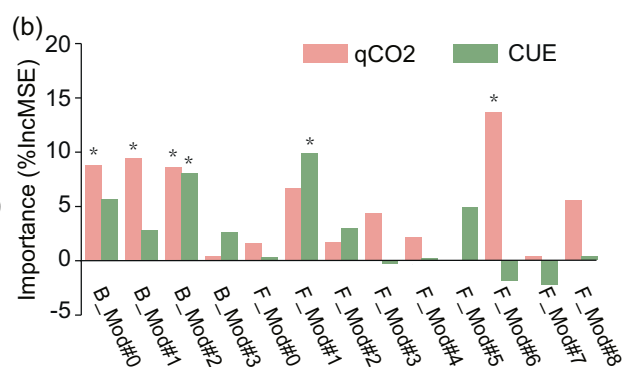

Microbial modules
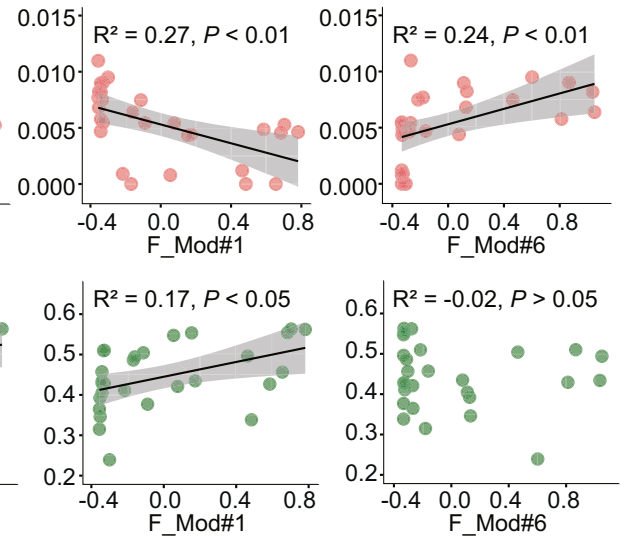

Fig. 3 Major bacterial and fungal assemblies explaining microbial metabolic efficiency. a Network diagram with nodes (OTUs) colored by each of the major ecological modules (assemblies) within co-occurrence network of bacterial and fungal community, respectively; b key bacterial and fungal assemblies predicting metabolic quotient $\left(\mathrm{qCO}_{2}\right)$ and carbon use efficiency (CUE) according to Random Forest analysis; c the relationships of key bacterial and fungal assemblies with metabolic efficiency. ${ }^{*} P<0.05 ;{ }^{* *} P<0.01$.

limitation, as illustrated by its negative relationships with altitude, soil C:nutrient ratios and the associated physiological traits. Therefore, the shift of bacterial assemblies from B_Mod\#2 to B_Mod\#0 with different trait strategies appears to be an important control on the increased C cost via maintenance and declined CUE with increasing altitude.

Similarly, shifts of fungal assemblies could also contribute to the variation in the metabolic efficiency along the altitude gradient. Microbial taxa within F_Mod\#6 may have slow-growing rates, as these taxa generally showed positive associations with $\mathrm{qCO}_{2}$, but negative relationships with CUE (Fig. 4b; Table S2). For instance, genus Mortierella, as the keystone genus for F_Mod\#6, were reported as essential psychrophiles and could produce high abundant trehalose and fatty acids to adapt to cold stress [51, 58]. In contrast, the keystone genus consisted of $F \_$Mod\#1, such as genera Neurospora was reported to have fast-growing lifestyles [59] and distributed mainly in warm regions like tropical and subtropical areas [60]. Overall, it is plausible that microbial taxa belonged to $B \_$Mod\#0 and F_Mod\#6 adapted to low temperature and resource quality conditions at higher altitude, but at the expense of more $C$ allocated to maintenance and lead to less efficient microbial community at higher altitude. Our findings link complex microbial community structure to functions under the framework of trait-based ecology, and highlight the importance of shifts in microbial functional groups with different traits for the metabolic efficiency of microbial community (Fig. 5).

While our study provides the empirical evidence for the roles of intrinsic properties of microbial community in regulating metabolic efficiency, these effects of biotic properties may be conflated with those of abiotic factors along the altitude gradient. For instance, declined soil $\mathrm{pH}$ significantly enhanced $\mathrm{qCO}_{2}$ and reduced microbial CUE, consistent with a recent study showing that acidic soils had very slow microbial growth rates and low CUE due to physiological constraints [61]. Moreover, higher $\mathrm{qCO}_{2}$ could also be interpreted as more soil $C$ (substrate) being available for microbial respiration at higher altitude [4]. However, it should be noted that biotic properties, particularly microbial resource acquisition traits and essential microbial assemblies, were important predictors of community-level metabolic efficiency even after considering these key abiotic factors. Therefore, our findings highlight that shifts in microbial assemblies with distinct life-history strategies should be incorporated into current trait-based models to better predict microbial community responses and the associated ecosystem functions under environmental changes (Fig. 5). Overall, microbial adaptions due to shifts in physiological traits and community composition lead to less efficient microbial community and lower energy investment into microbial growth and biomass production, which may further reduce the rates of essential ecosystem processes including soil organic matter decomposition and nutrient turnover at higher altitude.

\section{CONCLUSIONS}

To conclude, our results demonstrate that shifts in microbial assemblies with distinct physiological traits drive changes in community-level metabolic efficiency along the altitude gradient. At higher altitude, resource limitations drive microbial communities to invest more energy into resource acquisition strategies that trade off against growth yield, leading to higher metabolic quotient and lower CUE. These different microbial physiological traits could reflect the shifts in microbial functional groups with divergent strategies for coping with environmental constraints. We suggest that microbial taxa can adapt to nutrient limited and cold conditions at higher altitude, but at the expense of more maintenance efforts and lower metabolic efficiency. Our results highlight the shifts in trait-based strategies and microbial functional groups as essential factors regulating the metabolic efficiency of microbial community. This work could have important implications for $\mathrm{C}$ cycle in a changing world, and thus improve the predictive understanding of soil $\mathrm{C}$ responses to future climate change. 

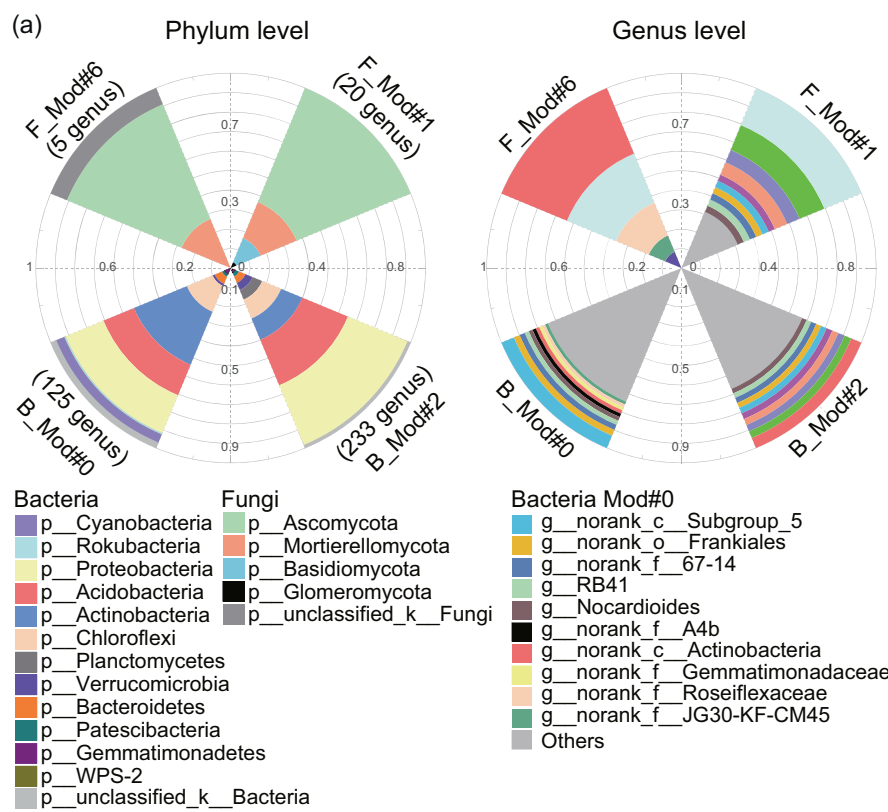

Fungi Mod\#1

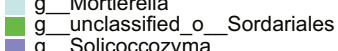

g_Solicoccozyma

g_unclassified_f_Chaetomiaceae

g_Paraglomus

g_Naganishia

g_Apiotrichum

g__Humicola

g_Epicoccum

Others

Fungi Mod\#6

g_unclassified_p_Ascomycota

g__Mortierella

g_Archaeorhizomyces

g_unclassified_k Fungi

Bacteria Mod\#0

Bacteria Mod\#2

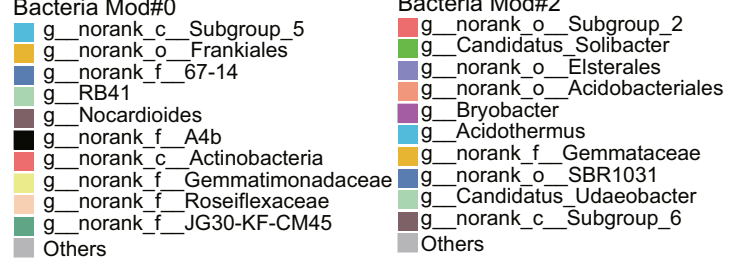

g_norank-o Frankiales

g_norank_o_Subgroup_2

g_- Candidatus Solibacter

g_norank_o_Elsterales

g_norank_o

- Acidothermu

_ norank___Gemmataceae

- Candidatus Udaer

Others

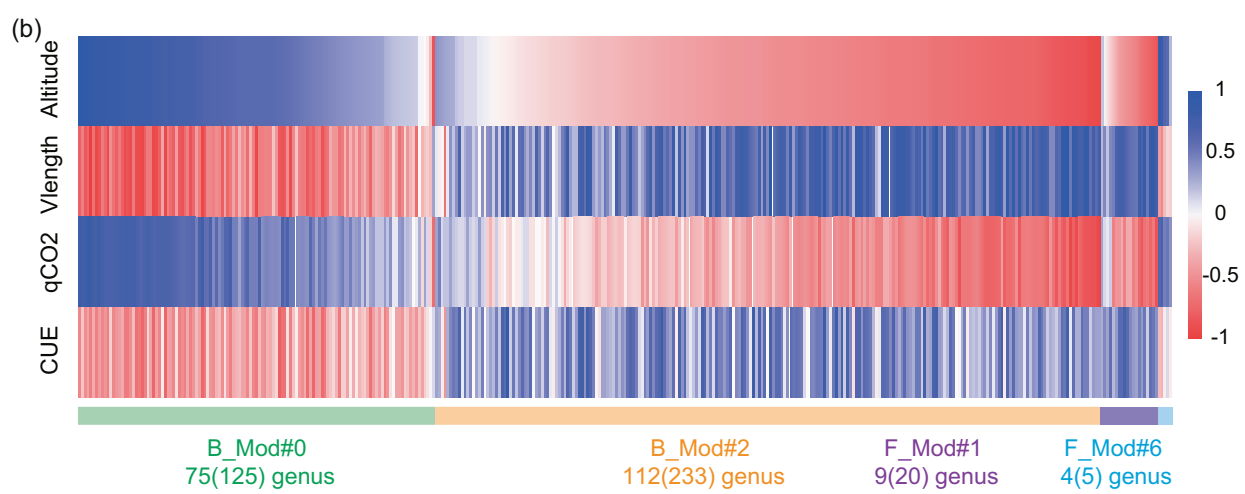

Fig. 4 Microbial taxa in key bacterial and fungal assemblies drive changes in microbial trait-based strategies and microbial metabolic efficiency. $\mathbf{a}$ The proportions of dominant phyla and genus in key bacterial and fungal assemblies; $\mathbf{b}$ relationships of the relative abundances of different genus in each key assemblies with microbial physiological traits and metabolic efficiency. B_Mod\#0 and B_Mod\#2 were key bacterial assemblies, and F_Mod\#1 and F_Mod\#6 were key fungal assemblies. Vlength, the relative C:nutrient-acquiring traits. The number of genus that correlated significantly $(P<0.05)$ with altitude in each key assemblies were shown. The number in parentheses indicates the total number of genus in each key assemblies.

(a) Environmental filters

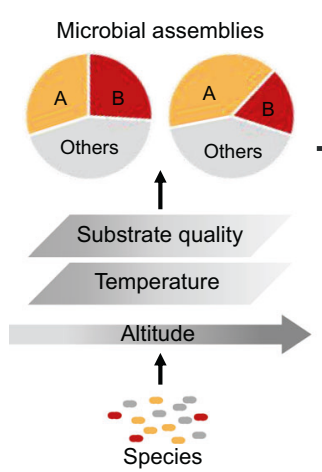

(b) Traits associated to adaptive assemblies (c) Trade-off in traits and its ecosystem consequences

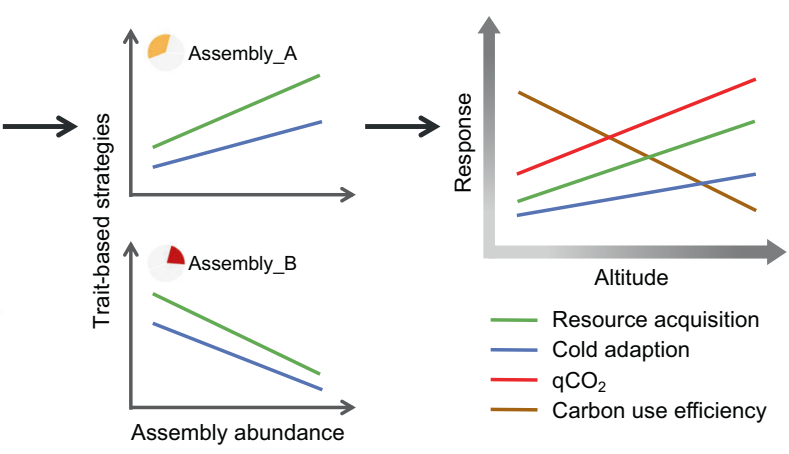

Fig. 5 A conceptual paradigm incorporating trait-based framework to predict metabolic efficiency under environmental changes. a Shift in microbial community composition under environmental changes; $\mathbf{b}$ changes in the proportion of microbial assemblies with distinct adaptive traits along the altitude gradient; $c$ trade-off in different microbial physiological traits and its consequences on metabolic efficiency at the community level. 


\section{REFERENCES}

1. Schimel J, Schaeffer S. Microbial control over carbon cycling in soil. Front Microbiol. 2012;3:1-11.

2. Liang C, Schimel JP, Jastrow JD. The importance of anabolism in microbial control over soil carbon storage. Nat Microbiol. 2017;2:17105.

3. Malik AA, Martiny JBH, Brodie EL, Martiny AC, Treseder KK, Allison SD. Defining trait-based microbial strategies with consequences for soil carbon cycling under climate change. ISME J. 2020;14:1-9.

4. Xu X, Schimel JP, Janssens IA, Song X, Song C, Yu G, et al. Global pattern and controls of soil microbial metabolic quotient. Ecol Monogr. 2017;87:429-41.

5. Chen L, Liu L, Mao C, Qin S, Wang J, Liu F, et al. Nitrogen availability regulates topsoil carbon dynamics after permafrost thaw by altering microbial metabolic efficiency. Nat commun. 2018;9:3951.

6. Wang C, Qu L, Yang L, Liu D, Morrissey E, Miao R, et al. Large-scale importance of microbial carbon use efficiency and necromass to soil organic carbon. Global Change Biol. 2021;27:2039-48.

7. Wieder WR, Bonan GB, Allison SD. Global soil carbon projections are improved by modelling microbial processes. Nat Clim Change. 2013;3:909-12.

8. Sinsabaugh RL, Manzoni S, Moorhead DL, Richter A. Carbon use efficiency of microbial communities: stoichiometry, methodology and modelling. Ecol Lett. 2013;16:930-9.

9. Xu M, Li X, Cai X, Gai J, Li X, Christie P, et al. Soil microbial community structure and activity along a montane elevational gradient on the Tibetan Plateau. Eur J Soil Biol. 2014;64:6-14.

10. Banerjee S, Walder F, Büchi L, Meyer M, Held AY, Gattinger A, et al. Agricultural intensification reduces microbial network complexity and the abundance of keystone taxa in roots. ISME J. 2019;13:1722-36.

11. Malik AA, Swenson T, Weihe C, Morrison EW, Martiny JBH, Brodie EL, et al. Drought and plant litter chemistry alter microbial gene expression and metabolite production. ISME J. 2020;14:2236-47.

12. Crowther TW, van den Hoogen J, Wan J, Mayes MA, Keiser AD, Mo L, et al. The global soil community and its influence on biogeochemistry. Science. 2019;365:eaav0550.

13. Nottingham AT, Bååth E, Reischke $S$, Salinas N, Meir P. Adaptation of soil microbial growth to temperature: Using a tropical elevation gradient to predict future changes. Global Change Biol. 2019;25:827-38.

14. Feng J, Wei K, Chen Z, Lü X, Tian J, Wang C, et al. Coupling and decoupling of soil carbon and nutrient cycles across an aridity gradient in the drylands of northern China: evidence from ecoenzymatic stoichiometry. Global Biogeochem Cycles. 2019;33:559-69.

15. Allison S, Weintraub M, Gartner T, \& Waldrop M. Evolutionary-economic principles as regulators of soil enzyme production and ecosystem function. In: Shukla $G$, Varma A., editors Soil enzymology. Soil Biology, vol 22. Berlin, Germany: Springer Berlin Heidelberg; 2011, pp 229-43.

16. Tribelli PM, López NI. Reporting key features in cold-adapted bacteria. Life. 2018;8:8.

17. Allison SD. A trait-based approach for modelling microbial litter decomposition. Ecol Lett. 2012;15:1058-70.

18. Fierer N, Bradford MA, Jackson RB. Toward an ecological classification of soil bacteria. Ecology. 2007;88:1354-64.

19. Li H, Yang S, Semenov MV, Yao F, Ye J, Bu R, et al. Temperature sensitivity of SOM decomposition is linked with a K-selected microbial community. Global Change Biol. 2021;27:2763-79.

20. Arce E, Archaimbault V, Mondy CP, Usseglio-Polatera P. Recovery dynamics in invertebrate communities following water-quality improvement: taxonomy- vs trait-based assessment. Freshw Sci. 2014;33:1060-73. 1014

21. Bench SR, llikchyan IN, Tripp HJ, Zehr JP. Two strains of crocosphaera watsonii with highly conserved genomes are distinguished by strain-specific features. Front Microbiol. 2011;2:261-261.

22. Ma B, Wang H, Dsouza M, Lou J, He Y, Dai Z, et al. Geographic patterns of cooccurrence network topological features for soil microbiota at continental scale in eastern China. ISME J. 2016;10:1891-901.

23. Du B, Kang H, Pumpanen J, Zhu P, Yin S, Zou Q, et al. Soil organic carbon stock and chemical composition along an altitude gradient in the Lushan Mountain, subtropical China. Ecol Res. 2014;29:433-9.

24. Yao T, Thompson L, Yang W. Different glacier status with atmospheric circulations in Tibetan Plateau and surroundings. Nat Clim Change. 2012;2:663-7.

25. Zhuo G, Ciren B, Wang J, Lan X. Analysis of regional climate characteristics of Tibetan herbal products growing on Mt. Seqilha. Resour Sci. 2010;32:1452-61.

26. Chen L, Flynn DFB, Zhang X, Gao X, Lin L, Luo J, et al. Divergent patterns of foliar $\delta 13 C$ and $\delta 15 \mathrm{~N}$ in Quercus aquifolioides with an altitudinal transect on the Tibetan Plateau: an integrated study based on multiple key leaf functional traits. J Plant Ecol. 2014;8:303-12.

27. Xu M, Wang G, Li X, Cai X, Li X, Christie P, et al. The key factor limiting plant growth in cold and humid alpine areas also plays a dominant role in plant carbon isotope discrimination. Front Plant Sci. 2015;3:961.
28. Du J, Gao R, Ma PF, Liu YM, Zhou KS. Analysis of stereoscopic climate features on Mt. Seqiha, Tibet. Plateau Mt Meteorol Res. 2009;19:14-18.

29. Hu Q-W, Wu Q, Cao G-M, Li D, Long R-J, Wang Y-S. Growing season ecosystem respirations and associated component fluxes in two alpine meadows on the Tibetan Plateau. J Integr Plant Biol. 2008;50:271-9.

30. IUSS Working Group. World reference base for soil resources 2006, first update 2007. World soil resources reports no.103. in World soil resources reports no. 103. Rome, Italy: FAO; 2007.

31. Walkley A. A critical examination of a rapid method for determining organic carbon in soils-effect of variations in digestion conditions and of inorganic soil constituents. Soil Sci. 1947;63:251-64.

32. Bray RH, Kurtz L. Determination of total, organic, and available forms of phosphorus in soils. Soil Sci. 1945;59:39-46.

33. Olsen SR, Cole CV, Watanabe FS. Estimation of available phosphorus in soils by extraction with sodium bicarbonate. Washington, DC: United States Department of Agriculture; 1954.

34. Liu YR, Delgado-Baquerizo M, Wang JT, Hu HW, Yang Z, He JZ. New insights into the role of microbial community composition in driving soil respiration rates. Soil Biol Biochem. 2018;118:35-41.

35. Yao Q, Liu J, Yu Z, Li Y, Jin J, Liu X, et al. Three years of biochar amendment alters soil physiochemical properties and fungal community composition in a black soil of northeast China. Soil Biol Biochem. 2017;110:56-67.

36. Quast C, Pruesse E, Yilmaz P, Gerken J, Schweer T, Yarza P, et al. The SILVA ribosomal RNA gene database project: improved data processing and web-based tools. Nucleic Acids Res. 2012;41:D590-D596.

37. Marx M-C, Wood M, Jarvis S. A microplate fluorimetric assay for the study of enzyme diversity in soils. Soil Biol Biochem. 2001;33:1633-40.

38. Moorhead DL, Sinsabaugh RL, Hill BH, Weintraub MN. Vector analysis of ecoenzyme activities reveal constraints on coupled $C, N$ and $P$ dynamics. Soil Biol Biochem. 2016;93:1-7.

39. Wardle DA, Ghani A. A critique of the microbial metabolic quotient (qCO2) as a bioindicator of disturbance and ecosystem development. Soil Biol Biochem. 1995;27:1601-10.

40. Wang Q, Liu S, Tian P. Carbon quality and soil microbial property control the latitudinal pattern in temperature sensitivity of soil microbial respiration across Chinese forest ecosystems. Glob Change Biol. 2018;24:2841-9.

41. Xu M, Li X, Kuyper TW, Xu M, Zhang J. High microbial diversity stabilizes the responses of soil organic carbon decomposition to warming in the subsoil on the Tibetan Plateau. Global Change Biol. 2021;27:2061-75.

42. Li Y, Lv W, Jiang L, Zhang L, Wang S, Wang Q, et al. Microbial community responses reduce soil carbon loss in Tibetan alpine grasslands under short-term warming. Global Change Biol. 2019;25:3438-49.

43. Vance $E$, Brookes $P$, Jenkinson D. An extraction method for measuring soil microbial biomass C. Soil Biol Biochem. 1987;19:703-7.

44. Sinsabaugh RL, Shah JJF. Ecoenzymatic stoichiometry and ecological theory. Annu Rev Ecol Evol S. 2012;43:313-43.

45. Cui $Y$, Wang X, Zhang X, Ju W, Duan C, Guo X, et al. Soil moisture mediates microbial carbon and phosphorus metabolism during vegetation succession in a semiarid region. Soil Biol Biochem. 2020;147:107814.

46. Breiman L. Random forests. Mach Learn. 2001;45:5-32.

47. Benjamini Y, Krieger AM, Yekutieli D. Adaptive linear step-up procedures that control the false discovery rate. Biometrika. 2006;93:491-507.

48. Delgado-Baquerizo M, Reith F, Dennis PG, Hamonts K, Powell JR, Young A, et al. Ecological drivers of soil microbial diversity and soil biological networks in the Southern Hemisphere. Ecology. 2018;99:583-96.

49. Cui Y, Moorhead DL, Guo X, Peng S, Wang Y, Zhang X, et al. Stoichiometric models of microbial metabolic limitation in soil systems. Glob Ecol Biogeogr. 2021;30:2297-311.

50. Nedwell DB. Effect of low temperature on microbial growth: lowered affinity for substrates limits growth at low temperature. Fems Microbiol Ecol. 1999;30:101-11.

51. Weinstein RN, Montiel PO, Johnstone K. Influence of growth temperature on lipid and soluble carbohydrate synthesis by fungi isolated from fellfield soil in the maritime Antarctic. Mycologia. 2000;92:222-9.

52. Varin T, Lovejoy $C$, Jungblut AD, Vincent WF, Corbeil J. Metagenomic analysis of stress genes in microbial mat communities from antarctica and the high arctic. Appl Environ Microb. 2012;78:549-59.

53. Nichols CM, Bowman JP, Guezennec J. Effects of incubation temperature on growth and production of exopolysaccharides by an antarctic sea ice bacterium grown in batch culture. Appl Environ Microbiol. 2005;71:3519-23.

54. Ren C, Zhang W, Zhong Z, Han X, Yang G, Feng Y, et al. Differential responses of soil microbial biomass, diversity, and compositions to altitudinal gradients depend on plant and soil characteristics. Sci Total Environ. 2018;610-1:750-8.

55. Kumar S, Suyal DC, Yadav A, Shouche Y, Goel R. Microbial diversity and soil physiochemical characteristic of higher altitude. PLoS ONE. 2019;14:e0213844. 
56. Ruuskanen MO, Colby G, St Pierre KA, St Louis VL, Aris-Brosou S, Poulain AJ. Microbial genomes retrieved from high arctic lake sediments encode for adaptation to cold and oligotrophic environments. Limnol Oceanogr. 2020;65:S233-S247.

57. Feng L-j, Jia R, Sun J-y, Wang J, Lv Z-h, Mu J, et al. Response of performance and bacterial community to oligotrophic stress in biofilm systems for raw water pretreatment. Biodegradation. 2017;28:231-44.

58. Robinson $\mathrm{CH}$. Cold adaptation in Arctic and Antarctic fungi. New Phytol. 2001;151:341-53.

59. Shahryari Z, Fazaelipoor M, Ghasemi Y, Lennartsson P, Taherzadeh M. Amylase and xylanase from edible fungus neurospora intermedia: production and characterization. Molecules. 2019;24:721.

60. Turner BC, Perkins DD, Fairfield A. Neurospora from natural populations: a global study. Fungal Genet Biol. 2001;32:67-92.

61. Malik AA, Puissant J, Buckeridge KM, Goodall T, Jehmlich N, Chowdhury S, et al. Land use driven change in soil $\mathrm{pH}$ affects microbial carbon cycling processes. Nat Commun. 2018;9:3591.

\section{ACKNOWLEDGEMENTS}

This study was supported by the National Natural Science Foundation of China (32071595, 41830756 and 42177022). We also thank the Fundamental Research Funds for the Central Universities (Program no. 2662019PY010 and 2662019QD055), Natural Science Fund of Hubei Province (2019CFA094), and the Strategic Priority Research Program (A) of the Chinese Academy of Sciences (Grant No. XDA20040502).

\section{AUTHOR CONTRIBUTIONS}

J.F. and Y.L. designed the study. X.M.Z. carried out the main experiments. J.F. analyzed the data with help from Y.L. and X.M.Z. J.F. wrote the first draft of the paper. Y.L., Q.Z., X.Q.Z. and Q.H. revised the paper. All authors reviewed the paper and approved the final version of the manuscript.

\section{COMPETING INTERESTS}

The authors declare no competing interests.

\section{ADDITIONAL INFORMATION}

Supplementary information The online version contains supplementary material available at https://doi.org/10.1038/s43705-021-00076-2.

Correspondence and requests for materials should be addressed to Yu-Rong Liu.

Reprints and permission information is available at http://www.nature.com/ reprints

Publisher's note Springer Nature remains neutral with regard to jurisdictional claims in published maps and institutional affiliations. Attribution 4.0 International License, which permits use, sharing, adaptation, distribution and reproduction in any medium or format, as long as you give appropriate credit to the original author(s) and the source, provide a link to the Creative Commons licence, and indicate if changes were made. The images or other third party material in this article are included in the article's Creative Commons licence, unless indicated otherwise in a credit line to the material. If material is not included in the article's Creative Commons licence and your intended use is not permitted by statutory regulation or exceeds the permitted use, you will need to obtain permission directly from the copyright holder. To view a copy of this licence, visit http://creativecommons. org/licenses/by/4.0/.

(c) The Author(s) 2021 